\title{
Role of calcium and AMP kinase in the regulation of mitochondrial biogenesis and GLUT4 levels in muscle
}

\author{
Edward O. Ojuka \\ Department of Human Biology, University of Cape Town, Cape Town 7700, South Africa
}

\begin{abstract}
Contractile activity induces mitochondrial biogenesis and increases glucose transport capacity in muscle. There has been much research on the mechanisms responsible for these adaptations. The present paper reviews the evidence, which indicates that the decrease in the levels of highenergy phosphates, leading to activation of AMP kinase (AMPK), and the increase in cytosolic $\mathrm{Ca}^{2+}$, which activates $\mathrm{Ca}^{2+}$ /calmodulin-dependent protein kinase (CAMK), are signals that initiate these adaptative responses. Although the events downstream of AMPK and CAMK have not been well characterized, these events lead to activation of various transcription factors, including: nuclear respiratory factors (NRF) 1 and 2, which cause increased expression of proteins of the respiratory chain; PPAR- $\alpha$, which up regulates the levels of enzymes of $\beta$ oxidation; mitochondrial transcription factor $\mathrm{A}$, which activates expression of the mitochondrial genome; myocyte-enhancing factor $2 \mathrm{~A}$, the transcription factor that regulates GLUT4 expression. The well-orchestrated expression of the multitude of proteins involved in these adaptations is mediated by the rapid activation of PPAR $\gamma$ co-activator (PGC) 1, a protein that binds to various transcription factors to maximize transcriptional activity. Activating AMPK using 5-aminoimidizole-4-carboxamide-1- $\beta$-D-riboside (AICAR) and increasing cytoplasmic $\mathrm{Ca}^{2+}$ using caffeine, W7 or ionomycin in L6 myotubes increases the concentration of mitochondrial enzymes and GLUT4 and enhances the binding of NRF-1 and NRF-2 to DNA. AICAR and Ca-releasing agents also increase the levels of PGC-1, mitochondrial transcription factor $\mathrm{A}$ and myocyte-enhancing factors $2 \mathrm{~A}$ and $2 \mathrm{D}$. These results are similar to the responses seen in muscle during the adaptation to endurance exercise and show that L6 myotubes are a suitable model for studying the mechanisms by which exercise causes the adaptive responses in muscle mitochondria and glucose transport.
\end{abstract}

\section{Mitochondrial biogenesis: GLUT4: $\mathrm{Ca}^{2+} /$ calmodulin-dependent protein kinase:} AMP kinase: Transcription factors

Regular physical exercise induces increases in the capacity of skeletal muscle to oxidize pyruvate and fatty acids (Holloszy \& Booth, 1976) and take up glucose (Ren et al. 1994). These adaptations enhance endurance performance and increase the capacity to expend energy. They also prevent and even reverse type 2 diabetes. The increased capacity for respiration, oxidation and energy expenditure are mediated by an increase in muscle mitochondria (Holloszy \& Booth, 1976), and the increase in glucose uptake capacity is, in part, the result of an increase in the content of GLUT4, the glucose transport protein (Ren et al. 1994). The present short review highlights the evidence, which indicates that the decrease in high-energy phosphates and the increase in intracellular $\mathrm{Ca}$ that occur during contractile activity trigger the processes that cause the adaptive increase in GLUT4 and mitochondria in muscle.

\section{Regulators of genes that express mitochondrial and GLUT4 proteins}

Mitochondria comprise gene products derived from nuclear and mitochondrial genomes. Each mitochondrion houses multiple copies of a small circular DNA (mitochondrial DNA) that contributes thirteen mRNA, twenty-two tRNA and two rRNA molecules (Hood, 2001). The thirteen mRNA molecules encode proteins of the respiratory chain. Transcription factors and other proteins required for

\footnotetext{
Abbreviations: AICAR, 5-aminoimidizole-4-carboxamide-1- $\beta$-D-riboside; AMPK, AMP kinase; $\mathrm{CAMK} \mathrm{Ca}^{2+} / \mathrm{calmodulin}^{-\mathrm{dependent}}$ protein kinase; NRF, nuclear respiratory factor; PCG-1, PPAR $\gamma$ co-activator-1. 
mitochondrial DNA transcription and mitochondrial assembly derive from nuclear genes. Mitochondrial biogenesis, therefore, requires an orchestrated expression of mitochondrial and nuclear genes. The regulation of this complex process is brought about by the interaction of various transcription factors and coactivators. The transcription factors that have been well characterized include nuclear respiratory factors (NRF) 1 and 2 and PPAR $\alpha$. PPAR- $\alpha$ is a member of a family of nuclear receptors that stimulate transcription of genes following activation by a ligand. These transcription factors are activated to bind specific DNA sequences when they interact with a coactivator of nuclear receptors termed PPAR $\gamma$ co-activator-1 (PGC-1; Puigserver et al. 1998; Wu et al. 1999; Scarpulla, 2002). Activation of NRF-1 by PGC-1 increases the expression of genes that encode mitochondrial respiratory chain proteins (Scarpulla, 2002) and 5-aminolevulinate synthase (Briadotti et al. 1993), as well as mitochondrial transcription factor $\mathrm{A}$, a regulator of mitochondrial DNA transcription and replication (Virbasius \& Scarpulla, 1994). Kelly's group (Vega et al. 2000) showed that activation of PPAR $\alpha$ by PGC-1 stimulates the expression of enzymes of fatty acid oxidation. Overexpression of PGC-1 greatly stimulates mitochondrial biogenesis in cultured myocytes and adipocytes (Puigserver et al. 1998; Wu et al. 1999). A single bout of swimming induces an increase in PGC-1 mRNA (Terada et al. 2001) and protein (Baar et al. 2002), as well as NRF-1 and NRF-2 binding to DNA (Baar et al. 2002). These observations provide support for the view that PGC-1, NRF-1, NRF-2 and PPAR $\alpha$ are involved in the stimulation of mitochondrial biogenesis by exercise and that activation of NRF-1, NRF-2 and PPAR $\alpha$ by PGC-1 results in a well-coordinated expression of mitochondrial proteins.

GLUT4 expression in response to exercise is regulated at the transcriptional level. Support for transcriptional activation comes from nuclear run-on transcription assays of nuclei from exercised muscles, which show that $30 \mathrm{~min}$ after the exercise bout transcription was unaltered, but increased by $1 \cdot 8$-fold after $3 \mathrm{~h}$, and returned to control values by $24 \mathrm{~h}$ (Neufer \& Dohm, 1993). GLUT4 gene transcription following exercise is regulated by a number of transcription factors, including myocyte-enhancing factor 2 and GLUT4-enhancing factor. It appears that full expression of GLUT4 after exercise occurs when both myocyte-enhancing factor 2 and GLUT4-enhancing factor are bound to their respective sites (Oshel et al. 2000).

\section{Signals that trigger the adaptive processes}

Some of the evidence that implicates changes in the concentrations of high-energy phosphates and $\mathrm{Ca}^{2+}$ as the initial events leading to the exercise-induced increase in mitochondrial biogenesis and GLUT4 up-regulation will be described.

\section{Changes in high-energy phosphates}

During muscle contraction ATP is hydrolysed to ADP by myosin ATPase to drive the cyclic association and dissociation of contractile proteins. Two reactions, driven by creatine kinase and adenylate kinase, act to replenish ATP by hydrolysing creatine phosphate and ADP to produce creatine and AMP respectively. Thus, during contractile activity of sufficiently high intensity ATP and creatine phosphate levels fall while the level of AMP rises. These changes in the levels of high-energy phosphates stimulate increases in GLUT4 and mitochondrial proteins. Support for this explanation comes from studies in which muscle creatine phosphate is depleted by feeding rats the creatine analogue $\beta$-guadinoproprionic acid. This treatment causes GLUT4 protein and mitochondrial enzymes (Bergeron et al. 2001) in muscle to increase.

A likely link between changes in high-energy phosphates and gene transcription is the enzyme AMP-activated protein kinase (AMPK). AMPK is activated during exercise and phosphorylates key target proteins that control flux through metabolic pathways (Winder \& Hardie, 1999). Activation of $\alpha_{2}$-AMPK can be simulated by administration of the drug 5-aminoimidizole-4-carboxamide-1- $\beta$-Driboside (AICAR) to animals. Injection of AICAR into rats activates AMPK and induces an increase in GLUT4 protein (Holmes et al. 1999). When epitroclearis muscles are incubated in vitro with AICAR for $18 \mathrm{~h}$ there is an increase in GLUT4 of approximately $50 \%$, demonstrating that activation of AMPK by AICAR is independent of systemic factors and that the increase in expression of GLUT4 in muscle is rapid (Ojuka et al. 2000). A subsequent study using L6 myotubes has shown that chronic treatment with AICAR for $5 \mathrm{~d}$ results in increases in GLUT4 and insulinstimulated glucose transport of approximately $60 \%$. The increase is prevented when adenosine-9-D-arabino-furanoside, an inhibitor of AMPK, is included in the medium (Ojuka et al. 2002b). The results of these studies and others (Hayashi et al. 1998; Zheng et al. 2001) provide strong evidence that AMPK is a key signalling intermediate that improves glucose transport by up regulating GLUT4 expression following exercise. Recently, Fryer et al. (2002) have demonstrated more directly, by overexpressing a constitutively-active AMPK gene in skeletal muscle, that AMPK improves glucose uptake and induces GLUT4 biogenesis.

Activation of AMPK also stimulates, at least in part, increases in mitochondrial proteins. After injection of rats with AICAR for 4 weeks there are marked increases in some (e.g. citrate synthase, succinate dehydrogenase and cytochrome c), but not all (e.g. carnitine palmitoyltransferase, hydroxyacetyl-CoA), mitochondrial enzymes in muscle (Winder et al. 2000). Chronic incubation of L6 myotubes with AICAR for $5 \mathrm{~d}$ increases 5-aminolevulinate synthase and cytochrome c protein by $30-50 \%$ compared with controls (Ojuka et al. 2002a).

The mechanism by which activated AMPK induces gene expression is not yet clear. As the enzyme is localized in the nucleus of many cells, it has been proposed that it directly regulates gene expression (Salt et al. 1998). It is also possible that gene expression occurs secondarily to AMPK activation. Support for the latter view comes from Bergeron et al. (2001), who have shown that chronic activation of AMPK by $\beta$-guadinoproprionic acid results in NRF-1 activation and mitochondrial biogenesis in muscle. 


\section{Increase in cytosolic calcium}

There is mounting evidence that the increase in cytosolic Ca triggered by each wave of sarcolemmal depolarization during muscle contraction also triggers the events that lead to mitochondrial biogenesis and increased GLUT4 expression. Lawrence \& Salsgiver (1983) have reported that exposure of primary cultures of fetal rat muscles to A-23187 $(3 \mu \mathrm{M})$, a Ca ionophore, induces increases in a number of mitochondrial proteins. Similarly, Freyssenet et al. (1999) have shown that continuous exposure of L6E9 myoblasts to A-23187 induces an increase in cytochrome c mRNA. As changes in high-energy phosphate were not measured in these experiments, there is the possibility that the effect of A-23187 on mitochondrial biogenesis was mediated by a decrease in ATP and an increase in AMP, resulting in activation of AMPK, rather than by $\mathrm{Ca}$ per se. Continuous exposure of cells to A-23187 decreases cell viability and number (E Ojuka and J Holloszy, unpublished results). Thus, because enzyme activity varies markedly between individual myotubes in primary cultures of rat skeletal muscle, it is possible that the sustained increase in cytosolic $\mathrm{Ca}$ may have selectively killed myotubes with a low mitochondrial content rather than stimulated mitochondrial biogenesis. Furthermore, because A-23187 mediates Ca entry through the sarcolemma and not from the sarcoplasmic reticulum, it is not clear whether these experiments mimicked the effects of exercise.

In order to address these questions a series of experiments have been conducted in which L6 myotubes were used and various agents that increase cytosolic $\mathrm{Ca}^{2+}$ by releasing $\mathrm{Ca}^{2+}$ from the sarcoplasmic reticulum (caffeine and W7) were compared with an ionophore (ionomycin) that allows $\mathrm{Ca}^{2+}$ entry through the sarcolemma. As L6 myotubes do not contract in response to an increase in cytosolic $\mathrm{Ca}^{2+}$, the generation of other potential signals induced by the breakdown of high-energy phosphates (increases in glycolytic intermediates, changes in redox state and $\mathrm{pH}$ etc.) that occur in contracting muscle were avoided. To improve cell viability and address the issue of selective destruction of myotubes with low mitochondrial content, myotubes were incubated intermittently for 3-5 h/d, instead of continuously, with the $\mathrm{Ca}^{2+}$-raising agents.

The results provide strong evidence that increases in cytosolic $\mathrm{Ca}^{2+}$, mediated either by intermittent release of $\mathrm{Ca}^{2+}$ from the sarcoplasmic reticulum or Ca entry through the sarcolemma, stimulate mitochondrial biogenesis and GLUT4 expression and that these effects are not mediated by a decrease in high-energy phosphate (Ojuka et al. $2002 a, b, 2003)$. Furthermore, in support of the role of $\mathrm{Ca}$, dantrolene, in concentrations that inhibit most of the increase in cytosolic $\mathrm{Ca}^{2+}$ induced by caffeine or $\mathrm{W} 7$, also inhibits the increases in mitochondrial and GLUT4 proteins. Similarly, removal of $\mathrm{Ca}^{2+}$ from the medium with [ethylenebis(oxonitrilo)] tetra-acetate attenuates both the increase in cytosolic $\mathrm{Ca}^{2+}$ and the stimulation of mitochondrial biogenesis by ionomycin. Data showing increases in PGC-1, NRF-1 and NRF-2, and mitochondrial transcription factor $\mathrm{A}$ in response to raising cytosolic $\mathrm{Ca}^{2+}($ Ojuka et al. 2003) provide evidence that the same mechanisms that mediate increases in GLUT4 and mitochondrial proteins in response to exercise also increase GLUT4 and mitochondrial proteins in L6 myotubes. This evidence shows that L6 myotubes are a good model for studying the adaptations in response to exercise.

As a preliminary step in the investigation of the pathway by which an increase in cytosolic $\mathrm{Ca}^{2+}$ stimulates mitochondrial biogenesis and GLUT4 expression, KN93, an inhibitor of $\mathrm{Ca}^{2+} /$ calmodulin-dependent protein kinase (CAMK; Corcoran \& Mean, 2001), was used to evaluate the possibility that the pathway activated by $\mathrm{Ca}^{2+}$ that leads to increased mitochondrial biogenesis involves CAMK. Inclusion of $10 \mu \mathrm{M}-\mathrm{KN} 93$ completely blocks the $\mathrm{Ca}^{2+}$-induced increases in GLUT4, representative proteins of the mitochondria and NRF-1, NRF-2 and myocyteenhancing factor 2 transcription factors (Ojuka et al. $2002 a, b, 2003)$. These results provide strong evidence that these $\mathrm{Ca}^{2+}$-induced effects on GLUT4 and proteins of the mitochondria involve CAMK. Further evidence in support of the role of CAMK have come from Williams' group (Wu et al. 2002), who have reported increases in mitochondrial density, cytochrome c and PGC-1 mRNA in transgenic mice that overexpress a constitutively active CAMK IV compared with mice that overexpress WT CAMK. Up-regulation of 5-aminolevulinate synthase, cytochrome c, and GLUT4 in L6 myotubes infected with adenovirus expressing a constitutively-active construct of CAMK IV has also been observed (E Ojuka and J Holloszy, unpublished results).

\section{Concluding remarks}

Evidence has been provided that shows that the adaptive increases in muscle GLUT4 and mitochondria induced by exercise are mediated by both the increase in cytosolic $\mathrm{Ca}^{2+}$, resulting in activation of CAMK, and the decrease in high-energy phosphates, resulting in activation of AMPK. On the basis of this observation, it seems reasonable to propose that during prolonged steady-state exercise that results in modest perturbations in the high-energy phosphate concentration and minimal activation of AMPK the primary adaptive stimulus is the increase in cytosolic $\mathrm{Ca}^{2+}$. On the other hand, intense training that involves periods of very strenuous exercise such as that used in interval training would, by activating both CAMK and AMPK, result in a more powerful stimulus to mitochondrial biogenesis and GLUT4 expression. This outcome would explain the greater effectiveness of high-intensity interval training compared with steady-state moderate-intensity exercise in inducing the adaptive response (Terada et al. 2001).

As AMPK and CAMK are closely-related enzymes that belong to the same protein kinase subfamily and recognize the same amino acid consensus sequence (Hawley et al. 1995), it seems possible that either CAMK or AMPK may activate the next step in the enzymic pathway leading to the stimulation of mitochondrial biogenesis and GLUT4 expression. As these adaptations are necessary for survival under conditions requiring prolonged vigorous exercise, it is not surprising that redundant mechanisms evolved for stimulating mitochondrial biogenesis and GLUT4 expression. 


\section{References}

Baar K, Wende AR, Jones TE, Marison M, Nolte LA, Chen M, Kelly DP \& Holloszy JO (2002) Adaptations of skeletal muscle to exercise: rapid increase in the transcriptional coactivator PGC-1. FASEB Journal 16, 1879-1886.

Bergeron R, Ren JM, Cadman KS, Moore IK, Perret P, Pypaert M, Young LH, Semenkovich CF \& Shulman GI (2001) Chronic activation of AMP kinase results in NRF-1 activation and mitochondrial biogenesis. American Journal of Physiology 281, E1340-E1346.

Briadotti G, Borthwick IA \& May BK (1993) Identification of regulatory sequences in the gene for 5 -aminolevulinate synthase from rat. Journal of Biological Chemistry 268, 1109-1117.

Corcoran EE \& Mean AR (2001) Defining $\mathrm{Ca}^{2+} /$ calmodulindependent protein kinase cascade in transcriptional regulation. Journal of Biological Chemistry 276, 2975-2978.

Freyssenet D, Di Carlo M \& Hood DA (1999) Calcium-dependent regulation of cytochrome $\mathrm{c}$ gene expression in skeletal muscle cells. Journal of Biological Chemistry 274, 9305-9311.

Fryer LG, Foufella F, Barnes K, Baldwin SA, Woods A \& Carling D (2002) Characterization of the role of the AMPactivated protein kinase in the stimulation of glucose transport in skeletal muscle cells. Biochemical Journal 363, 167-174.

Hawley SA, Selbert MA, Goldstein EG, Edelman AM, Carling D \& Hardie DG (1995) 5'-AMP activated protein kinase cascade, and $\mathrm{Ca}^{2+} /$ calmodulin activates the calmodulin-dependent protein kinase I kinase, via three independent mechanisms. Journal of Biological Chemistry 270, 27186-27191.

Hayashi T, Hirshman MF, Kurth EJ, Winder WW \& Goodyear LJ (1998) Evidence for 5' AMP-activated protein kinase mediation of the effect of muscle contraction on glucose transport. Diabetes 49, 1369-1373.

Holloszy JO \& Booth FW (1976) Biochemical adaptations to endurance exercise in muscle. Annual Review of Physiology 38, 273-291.

Holmes BF, Kurth-Kraczek EJ \& Winder WW (1999) Chronic activation of 5'-AMP-activated protein kinase increases GLUT-4, hexokinase, and glycogen in muscle. Journal of Applied Physiology 87, 1990-1995.

Hood DA (2001) Plasticity in skeletal, cardiac, and smooth muscle. Contractile activity-induced mitochondrial biogenesis in skeletal muscle. Journal of Applied Physiology 90, 1137-1157.

Lawrence JC Jr \& Salsgiver WJ (1983) Levels of enzymes of energy metabolism are controlled by activity of cultured rat myotubes. American Journal of Physiology 244, C348-C355.

Neufer PD \& Dohm GL (1993) Exercise induces a transient increase in transcription of the GLUT-4 gene in skeletal muscle. American Journal of Physiology 265, C1597-C1603.

Ojuka EO, Jones TE, Han DH, Chen M \& Holloszy JO (2003) Raising $\mathrm{Ca}^{2+}$ in L6 myotubes mimics effects of exercise on mitochondrial biogenesis in muscle. FASEB Journal 17, 675-681.

Ojuka EO, Jones TE, Han DH, Chen M, Wanhoff BR, Sturek M \& Holloszy JO (2002a) Intermittent increases in cytosolic $\mathrm{Ca}^{2+}$ stimulate mitochondrial biogenesis in muscle cells. American Journal of Physiology 283, E1040-E1045.

Ojuka EO, Jones TE, Nolte LA, Chen M, Wonhoff BR, Sturek M \& Holloszy JO (2002b) Regulation of GLUT-4 biogenesis: evidence for involvement of AMPK and $\mathrm{Ca}^{2+}$. American Journal of Physiology 282, E1008-E1013.
Ojuka EO, Nolte LA \& Holloszy JO (2000) Increased expression of GLUT-4 and hexokinase in rat epitroclearis muscle exposed to AICAR in vitro. Journal of Applied Physiology 88, 1072-1075.

Oshel KM, Knight JB, Cao KT, Thai MV \& Olson AL (2000) Identification of a 30 base pair regulatory element and a novel DNA binding protein that regulates the human GLUT-4 promoter in transgenic mice. Journal of Biological Chemistry 275, 23666-23673.

Puigserver P, Wu Z, Park CW, Graves R, Wright $\mathrm{M}$ \& Spiegelman BM (1998) A cold-inducible coactivator of nuclear receptors linked to adaptive thermogenesis. Cell 92, 829-839.

Ren JM, Semenkovich CF, Gulve EA, Gao EA \& Holloszy JO (1994) Exercise induces rapid increases in GLUT-4 expression, glucose transport capacity, and insulin-stimulated glycogen storage in muscle. Journal of Biological Chemistry 269, 14396-14401.

Salt I, Celler JW, Hawley SA, Prescott A \& Woods A (1998) AMP-activated protein kinase: greater AMP dependence, and preferential nuclear localization, of complexes containing alpha2 isoform. Biochemical Journal 334, 177-187.

Scarpulla RC (2002) Nuclear activators and coactivators in mammalian mitochondrial biogenesis. Biochimica et Biophysica Acta 1576, 1-14.

Terada S, Yokozeki T, Kawanaka K, Ogawa K, Higuchi M, Ezaki O \& Tabata I (2001) Effects of high-intensity swimming training on GLUT-4 and glucose transport activity in rat skeletal muscle. Journal of Applied Physiology 90, 2019-2024.

Vega R, Huss JM \& Kelly DP (2000) The coactivator PGC-1 cooperates with peroxisome proliferator-activated receptor $\alpha$ in transcriptional control of nuclear genes encoding mitochondrial fatty acid oxidation enzymes. Molecular and Cellular Biology 20, 1868-1876.

Virbasius JV \& Scarpulla RC (1994) Activation of the human transcription factor A gene by nuclear respiratory factors: a potential regulatory link between nuclear and mitochondrial gene expression in organelle biogenesis. Proceedings of the National Academy of Sciences USA 91, 1309-1313.

Winder WW \& Hardie DG (1999) AMP-activated protein kinase, a metabolic master switch: possible roles in type 2 diabetes. American Journal of Physiology 277, E1-E10.

Winder WW, Holmes BF, Rubink DS, Jensen EB, Chen M \& Holloszy JO (2000) Activation of AMP-activated protein kinase increases mitochondrial enzymes in skeletal muscles. Journal of Applied Physiology 88, 2219-2226.

Wu H, Kanatous SB, Thumond FA, Gallardo T, Isotani E, BasselDurby R \& Williams RS (2002) Regulation of mitochondrial biogenesis in skeletal muscle in CAMK. Science 296, 349-352.

Wu Z, Puisgsever P, Anderson U, Zhang C, Adelmant G, Moother V, Troy A, Cinti S, Lowell B, Scarpulla RC \& Speigelman BM (1999) Mechanisms controlling mitochondrial biogenesis and respiration through the thermogenic coactivator PGC-1. Cell 98, 115-124.

Yaspelkis BB, Castle AL, Farrar RP \& Ivy JL (1997) Contraction-induced intercellular signals and their relationship to muscle GLUT-4 concentration. American Journal of Physiology 272, E118-E125.

Zheng D, MacLean PS, Pohnert SC, Knight JB, Olson AL, Winder WW \& Dhom GL (2001) Regulation of muscle GLUT-4 transcription by AMP-activated protein kinase. Journal of Applied Physiology 91, 1073-1083. 